\title{
Developing a measure for health professionals' attitudes toward veterans
}

\author{
Sarah Knopf-Amelung*1 ${ }^{*}$, Margaret Brommelsiek ${ }^{1}$, Jane Anthony Peterson ${ }^{1}$, Zack Roman ${ }^{2}$, Tracy Lynn Graybill ${ }^{1}$ \\ ${ }^{1}$ School of Nursing and Health Studies, University of Missouri-Kansas City, Kansas City, MO, United States \\ ${ }^{2}$ University of Kansas, Lawrence, KS, United States
}

Received: January 19, 2018

DOI: $10.5430 /$ jnep.v8n7p60
Accepted: February 22, $2018 \quad$ Online Published: February 28, 2018

URL: https://doi.org/10.5430/jnep.v8n7p60

\begin{abstract}
U.S. veterans have complex healthcare needs that require professionals who are properly trained to address these issues. However, little is known about the attitudes that nurses and other professionals have toward veteran patients, particularly those working in community-based settings where it is unlikely training on veterans' issues has occurred. Understanding health professionals' attitudes toward caring for veterans is an important step in developing a workforce that is knowledgeable and willing to serve this complex and growing population. The purpose of this study was to develop and validate the Health Professionals' Attitudes Toward Veterans (HPATV) scale, which explores attitudes regarding military cultural sensitivity and awareness, provision of care to veteran patients, and the prominent veterans' health issues. The HPATV was developed across several phases, including review of existing measures and literature regarding veterans' health and attitude structure, hypothesis of a factor structure, identification of a theoretical framework for attitude construction, item generation, 3-round Delphi survey to refine items and test content validity, piloting the measure among health professions students, and exploratory (EFA) and confirmatory factor analysis (CFA). Following CFA, the final 14-item scale revealed 3 latent factors to describe health professionals' more nuanced attitudes toward working with veteran patients: culture, care, and health. The HPATV is a validated and readily available tool for needs assessment, quality improvement, and evaluation. Use of this tool will help increase understanding of these culture, care, and health domains and generate quality improvement initiatives based on them-ultimately benefiting veteran patients through more sensitive, patient-centered care.
\end{abstract}

Key Words: Veterans health, Healthcare provider attitudes, Delivery of health care, Cultural competency, Evaluation tool

\section{INTRODUCTION}

In the U.S., 1 in every 15 persons is a veteran. ${ }^{[1]}$ Military service can have a long-term psychological, physical, and socioeconomic impact on veterans and their families. ${ }^{[2]}$ Veterans have higher rates of multiple chronic conditions and report a lower health-related quality of life, including worse mental and physical health status, as compared to non-veterans. ${ }^{[3-5]}$ Therefore, it is imperative that health systems and profes- sionals are prepared to treat the complex needs of veterans. However, little is known about the attitudes that nurses and other healthcare providers have toward veteran patients, particularly those working in community-based settings where it is unlikely training on veterans' experiences and health issues has occurred.

Of the 22 million U.S. veterans, less than 9 million receive care from the U.S. Department of Veterans Affairs (VA)

\footnotetext{
${ }^{*}$ Correspondence: Sarah Knopf-Amelung; Email: knopfsm@umkc.edu; Address: School of Nursing and Health Studies, University of MissouriKansas City, 2464 Charlotte St., Kansas City, MO 64108, United States.
} 
healthcare system. ${ }^{[2]}$ The VA system provides a comprehensive package of services, yet an increasing number of veterans utilize civilian services due to perceptions of increased flexibility, accessibility, and confidentiality; fear of stigma toward behavioral health issues; and living in a rural area without access to a VA facility. ${ }^{[6-9]}$ Passage of the Veterans Access, Choice and Accountability Act of 2014 further supported utilization of community-based services by allowing veterans to receive care from eligible non-VA providers if they meet certain requirements. ${ }^{[10]}$ The number of veterans seeking community-based care is projected to grow, ${ }^{[6,7,11-13]}$ shifting even more responsibility to community-based settings and increasing the demand for qualified professionals. ${ }^{[14]}$

Unfortunately, many veterans utilizing community healthcare services have never been identified as veterans and providers are unable to associate the presenting health issues as being related to their military service. ${ }^{[1,2]}$ Limited data are available regarding health professionals' knowledge of veteran health needs and their interest in increasing knowledge and skills to better serve this population. ${ }^{[15,16]}$ One study of 300 behavioral health and primary care providers found that only a third had received VA-related training, less than half screened patients for military service, and less than $30 \%$ felt knowledgeable about how to refer to the VA health system. ${ }^{[15]}$ A similar study of clinicians in behavioral health settings found providers had limited training or supervision in veteran-specific care. ${ }^{[6]}$ A 2012 survey showed just 3 out of 110 U.S. medical schools included education about veterans. ${ }^{[17]}$ These gaps in education impede the provision of patient-centered care for veterans in community clinics.

Often connected with a deficit in knowledge and experience, healthcare professionals' attitudes toward their patients can affect the quality of care provided. ${ }^{[18]}$ A culturally competent attitude - one that avoids prejudice, bias, or stereotyping — can help reduce disparities in healthcare. ${ }^{[19]}$ Providers' culturally aware attitudes can aid in the early identification of health issues related to military service and help build patient trust. ${ }^{[6]}$ A study on veteran disclosure of trauma to health providers found that veterans do not disclose their experiences because of perceptions that providers are not interested in their experiences or do not believe them. ${ }^{[20]}$

Little is known about the attitudes that nurses and other health professionals have about veteran patients. Existing scales have explored the general public's attitude toward veterans, and health care professionals' attitudes toward other patient sub-populations, such as persons experiencing homelessness, persons with disabilities, older adults, and the LGBTQI population. ${ }^{[21-23]}$ The purpose of this study was to develop and

Published by Sciedu Press validate a measure that explored health professionals' attitudes regarding military cultural sensitivity and awareness, provision of care to veteran patients, and the prominent veterans' health issues. This scale will help assess existing attitudes and inform efforts to increase workforce capacity to care for veterans. This paper describes the process of developing and validating the measure and implications for how it can be used.

\section{METHODS}

The Health Professionals' Attitudes toward Veterans (HPATV) scale was developed across several phases, including review of existing measures and literature regarding veterans' health and attitude structure, hypothesis of a factor structure, identification of a theoretical framework for attitude construction, item generation, Delphi survey to refine items and test content validity, piloting the measure among health professions students, and exploratory and confirmatory factor analysis to test construct validity and confirm factor structure.

\subsection{Item generation}

A literature search was conducted to identify existing measures of health professionals' attitudes toward veterans and topics of importance regarding military culture and veterans' health. One existing measure was identified, but it focused on personal beliefs outside the context of healthcare. ${ }^{[24]}$

The tripartite (or multicomponent) model of attitudes ${ }^{[25-27]}$ was selected to construct the HPATV items. Broadly defined, attitudes are psychological tendencies, which are measured by the degree to which a person favors or disfavors a specific object. ${ }^{[25]}$ Within this framework, there are 3 components of attitude: affective, cognitive, and behavioral. ${ }^{[28]}$ The HPATV scale seeks to explore the cognitive, affective, and behavioral components of health professionals' attitudes about working with veteran patients. The cognitive component encompasses beliefs and knowledge. ${ }^{[25]}$ Beliefs are the linkages or associations of thoughts about the object. ${ }^{[25,29]}$ Cognition is a compilation of ideas, knowledge, and beliefs about an attitude object. ${ }^{[30]}$ Affects are feelings, mood, or temperament generally associated with a particular attitude object. ${ }^{[30,31]}$ Affect is influenced not only by what is known about the attitude object, but can also be influenced by experience or inexperience with the object. ${ }^{[30]}$ Affect is also associated with the level of strength of beliefs about the object. ${ }^{[30]}$ Lastly, the behavioral component of attitude relates to how one performs an action when confronted with the attitude object. $^{[30]}$

A 3-factor structure was hypothesized to describe the attitudes health professionals have toward veteran patients. 
These factors included military cultural sensitivity and awareness (culture), providing care to veteran patients (care), and views toward prominent veterans' health issues (health). Items were generated based on these domains and address cognitive, affective, and behavioral components. The initial version of the scale contained 73 Likert-type items with a 5-choice format (1-strongly disagree, 2-disagree, 3-neutral, 4-agree, 5-strongly agree).

\subsection{Item refinement}

A 3-round Delphi survey was used to refine item construction, establish content validity, and reach consensus on the scale's items. A Delphi survey is an iterative, structured feedback process that utilizes several rounds of surveying content experts to develop consensus. ${ }^{[32]}$ This method has been used previously to develop and validate attitudinal scales, including scales designed to measure health professions students' attitudes about professionalism and homelessness. ${ }^{[21,33]}$ The process of this Delphi survey was modeled after the methods used by Klemenc-Ketis \& Vrecko ${ }^{[33]}$ and conducted via online surveys. Procedures were approved by the university's institutional review board.

A convenience sample of participants was recruited through university faculty and local VA contacts. The first round of the Delphi survey, participants were asked whether each item should be part of the scale, should not be part of the scale, or should be part of the scale but needed revising (open-ended comments were provided to capture suggested revisions for each item). Based on aggregate feedback from participants, including a minimum threshold of $80 \%$ consensus, a revised scale was developed. In the second round, participants were asked to rate each item for 1) clarity of assessment of attitudes and 2) necessity for assessment of attitudes. Based on aggregate feedback from participants with an $80 \%$ consensus threshold, a revised scale was developed. In the third and final round, participants provided a yes/no response to approve inclusion of each item in the final version. Based on aggregate feedback from participants with an $80 \%$ consensus threshold, a final version of the scale was developed.

\subsection{Piloting of measure}

The measure was piloted with a convenience sample of graduate-level health professions students (Doctor of Nursing Practice [DNP], Doctor of Clinical Psychology [Clinical Psychology PhD], Doctor of Pharmacy [PharmD], and Master of Social Work [MSW] students) participating in an advanced nursing education interprofessional immersion course and clinical experience at the local VA that focused on improving care to medically underserved veterans with multiple chronic conditions. Students completed the scale pre- and post-course.

\subsection{Exploratory and confirmatory factor analysis}

Exploratory and Confirmatory Factor Analysis (EFA and CFA) were conducted using the $\mathrm{R}$ programming environment and the $\mathrm{R}$ packages: lavaan, semPlot, foreign, psych, and pander. ${ }^{[34-39]}$ Bivariate correlations were explored to find redundant items; none were found to be problematic ( $r$ $>0.8$ ). After this stage, an EFA was conducted using the first wave of data (pre-course) to explore the factor loading structure of 2, 3, and 4 factor solutions. The 3-factor solution was selected and an iterative procedure of CFAs were conducted, resulting in the final 3-factor solution, where latent factors represent culture, care, and health. The accepted model was then verified by testing it again with the second wave of data (post-course).

\section{RESUlTS}

\subsection{Delphi survey}

Eighteen participants agreed to take part in the Delphi survey and were invited to participate in all rounds, although some did not complete the survey in all rounds (15 participated in round 1,15 in round 2 , and 13 in round 3 ). Participants were healthcare professionals at the local VA or faculty at University of Missouri-Kansas City. Participants' professional backgrounds included advanced practice nursing, clinical psychology, pharmacy, social work, and research/evaluation. Two participants were also veterans. In the first round, 41 of 73 original items $(56.1 \%)$ were retained; 32 of 41 items (78.0\%) were retained in the second round; and 31 of 32 items $(96.9 \%)$ were retained in the final round.

\subsection{Piloting of measure}

Across 4 semester cohorts, 54 DNP $(n=22)$, clinical psychology $\mathrm{PhD}(\mathrm{n}=10)$, PharmD $(\mathrm{n}=9)$, and MSW $(\mathrm{n}=13)$ students participated in the 8-week course and completed the 31 -item measure at pre- and post-course. The majority were white $(71.7 \%)$ and female $(83.0 \%)$. The average age was 30.78 and ranged from 23 to 54 years of age. Table 1 contains the means and standard deviations at pre- and post-course. Items 3, 14, 15, 23, 24, 26, and 27 were reversecoded to indicate that a higher mean corresponded with a positive attitude.

\subsection{Preparation and missing data techniques}

Graphical exploration of the data identified 2 data entry errors that were consequently resolved (i.e., 44 instead of 4). Reverse items were recoded. Missing data was explored and found to be non-problematic (i.e., missing at random). Between the 2 measurement occasions, a total of 15 of 3162 responses were missing (this proportion only included items 
relevant to the CFA and does not include missing demo- relation matrices provided to both the EFA and CFA were graphic information). To deal with missing data, the cor- calculated using only pairwise complete observations.

Table 1. HPATV item pre- and post-course means and standard deviations

\begin{tabular}{|c|c|c|c|c|c|}
\hline \multirow{2}{*}{\multicolumn{2}{|c|}{ HPATV Item }} & \multicolumn{2}{|c|}{$\begin{array}{l}\text { Pre-Course } \\
\mathrm{n}=54\end{array}$} & \multicolumn{2}{|c|}{$\begin{array}{l}\text { Post-Course } \\
\text { n }=54\end{array}$} \\
\hline & & \multirow{2}{*}{$\begin{array}{l}\mathbf{M} \\
2.47\end{array}$} & \multirow{2}{*}{$\begin{array}{l}\text { SD } \\
1.08\end{array}$} & \multirow{2}{*}{$\begin{array}{l}\mathbf{M} \\
3.86\end{array}$} & \multirow{2}{*}{$\begin{array}{l}\text { SD } \\
.601\end{array}$} \\
\hline 1 & $\begin{array}{l}\text { I have sufficient knowledge of differences between military eras (e.g., Vietnam era vs. } \\
\text { Iraq/Afghanistan era) that may impact Veterans’ health. }\end{array}$ & & & & \\
\hline 2 & $\begin{array}{l}\text { I routinely ask Veterans about the community-based services they may need (e.g., housing, } \\
\text { vocational services). }\end{array}$ & 2.73 & 1.13 & 3.82 & .962 \\
\hline 3 & $\begin{array}{l}\text { I am uncomfortable talking to Veterans about possible symptoms of post-traumatic stress } \\
\text { disorder (PTSD) including nightmares and feeling on guard. }\end{array}$ & 3.65 & 1.23 & 3.88 & 1.28 \\
\hline 4 & I am knowledgeable about behavioral health issues prevalent in the Veteran population. & 3.39 & 1.00 & 4.31 & .583 \\
\hline 5 & I am comfortable discussing alcohol and drug use with Veteran patients. & 3.74 & 1.05 & 4.43 & .575 \\
\hline 6 & Veterans have unique health needs. & 4.49 & .644 & 4.75 & .440 \\
\hline 7 & I routinely screen patients for a history of military service. & 2.75 & 1.11 & 3.66 & 1.06 \\
\hline 8 & I am comfortable addressing the unique health needs of women Veterans. & 3.45 & 1.14 & 3.98 & .735 \\
\hline 9 & $\begin{array}{l}\text { I am comfortable asking a Veteran for permission to discuss health issues related to their } \\
\text { military service. }\end{array}$ & 3.8 & .987 & 4.45 & .503 \\
\hline 10 & It is necessary to ask a Veteran permission to discuss their military service. & 4.37 & .774 & 4.53 & .612 \\
\hline 11 & I feel confident in my ability to recognize the symptoms of traumatic brain injury (TBI). & 3.00 & 1.00 & 3.71 & .756 \\
\hline 12 & I have sufficient knowledge of Veterans’ health needs. & 2.82 & .974 & 4.04 & .605 \\
\hline 13 & $\begin{array}{l}\text { I feel comfortable asking Veterans about sexual harassment or sexual trauma they may have } \\
\text { experienced while serving in the military. }\end{array}$ & 3.08 & 1.11 & 3.88 & .864 \\
\hline 14 & Working with Veteran patients feels more challenging than working with non-Veteran patients. & 3.12 & 1.09 & 3.10 & 1.13 \\
\hline 15 & I need more training on military culture to feel comfortable working with Veterans. & 2.18 & 1.07 & 3.22 & .966 \\
\hline 16 & $\begin{array}{l}\text { I have sufficient knowledge of post-traumatic stress disorder (PTSD) triggers that could occur in } \\
\text { a Veteran's daily life. }\end{array}$ & 3.22 & 1.03 & 4.00 & .800 \\
\hline 17 & I feel comfortable having a conversation with Veterans about their military experience. & 3.90 & .922 & 4.45 & .541 \\
\hline 18 & I routinely screen Veterans for behavioral health issues prevalent in this population. & 2.78 & 1.17 & 3.90 & .953 \\
\hline 19 & $\begin{array}{l}\text { I am confident in my ability to screen Veterans for environmental exposures (e.g., Agent } \\
\text { Orange, toxic nerve agents). }\end{array}$ & 2.18 & .888 & 3.14 & 1.05 \\
\hline 20 & $\begin{array}{l}\text { It is important for health professionals to understand military culture in order to provide quality } \\
\text { care to Veterans. }\end{array}$ & 4.65 & .559 & 4.65 & .483 \\
\hline 21 & Military sexual trauma is an important issue to consider among Veteran patients. & 4.53 & .612 & 4.78 & .415 \\
\hline 22 & I plan to stay current on the literature regarding Veteran health issues. & 4.02 & .707 & 4.32 & .768 \\
\hline 23 & The health needs of Veterans are no different than those of non-Veterans. & 4.04 & .720 & 3.84 & .925 \\
\hline 24 & I am not familiar with the resources and services available to Veterans. & 2.82 & 1.09 & 3.78 & .789 \\
\hline 25 & $\begin{array}{l}\text { It is important to let Veterans decide if and how they share their military experiences with health } \\
\text { professionals. }\end{array}$ & 4.35 & .744 & 4.49 & 612 \\
\hline 26 & I feel more comfortable working with non-Veteran patients than Veteran patients. & 3.08 & .997 & 3.53 & .946 \\
\hline 27 & Sometimes I feel I do not relate well to Veteran patients due to my lack of military experience. & 2.75 & 1.29 & 3.27 & 1.10 \\
\hline 28 & I try not to make assumptions about how a Veteran views their military experience. & 4.33 & .554 & 4.35 & .779 \\
\hline 29 & I make an effort to understand the needs of Veterans. & 4.31 & .648 & 4.72 & .454 \\
\hline 30 & $\begin{array}{l}\text { I try to consider the trauma that may have occurred before and/or after a Veteran's military } \\
\text { experience. }\end{array}$ & 4.31 & .648 & 4.62 & .490 \\
\hline & I am interested in increasing my knowledge of Veterans’ needs. & 4.82 & .434 & 4.59 & .572 \\
\hline
\end{tabular}

*Items 4, 6, 8, 10-12, 14, 17-21, 23, 25, 29-31 were excluded from HPATV after confirmatory factor analysis 


\subsection{Exploratory factor analysis}

An EFA was conducted on the first wave of data to explore the number of likely factors in the items. Further, this stage helped identify which items may have problematic cross loadings (i.e., loadings above .3 on multiple factors). The first stage explored the eigenvalue structure of the model utilizing a scree plot. The plot suggested the most likely solution was 2, 3, or 4 factors based on elbow rule and the Keiser-Guttman criteria. ${ }^{[40,41]}$ Thus, 2, 3, or 4 factor solutions were generated utilizing varimax (orthogonal) rotations. The 3 factor solution provided the least cross loadings, and was thus selected.

\subsection{Confirmatory factor analysis}

\subsubsection{CFA as an iterative procedure}

The final accepted model was selected through an iterative process:

(1) A model was specified and the CFA was conducted.

(2) The model fit was checked by comparing fit statistics (RMSEA, SRMR, CFI, and TLI) to accepted thresholds for acceptable model fit (RMSEA \& SRMR $<.08$, and CFI \& TLI $>.90^{[42]}$ ).

(3) If model fit was unacceptable, problematic items were removed and the process was repeated until good model fit was obtained. Problematic items were characterized by having low loadings. Further issues arose if inspection of modification indices indicated that model fit would significantly improve from adding cross loadings.

(4) Once a final model was selected, the model was tested on a new wave of data to verify the model was not over-fit (over-fitting refers to a model only performing well on a specific sample).

\subsubsection{Model building}

The following factor model was specified: Factor 1 (culture) items: 1, 15, 20, 25, 27, 28. Factor 2 (care) items: 2, 6, 7, $8,9,10,14,17,22,23,24,26,29,31$. Factor 3 (health) items: $3,4,5,11,12,13,16,18,19,21,30$. No cross loadings or correlated error terms were specified; however, the 3 factors were permitted to correlate. The original model converged, however the model implied covariance matrix was non-positive definite. Exploration of this matrix revealed a non-positive variance also known as a Heywood case. ${ }^{[33]}$ Thus, the original model was rejected. Problematic items were removed and the model was re-specified. This iterative procedure was then completed 17 times resulting in the final model ( 17 items were removed). Figure 1 displays the final 14 items by factor.

\subsubsection{Final model and verification of results}

Inspection of model fit indices revealed acceptable to good model fit: $\mathrm{RMSEA}=0.025, \mathrm{SRMR}=0.079, \mathrm{CFI}=0.985$, $\mathrm{TLI}=0.981$. The standardized factor loading structure is presented in Table 2. To verify the model did not over-fit the sample, it was tested on the second wave of data. The model performed well, obtaining acceptable to good fit: RM$\mathrm{SEA}=0.023, \mathrm{SRMR}=0.075, \mathrm{CFI}=0.983, \mathrm{TLI}=0.991$. Table 2 also provides the standardized solution, including the factor loading structure. Further, a Chi Squared difference test comparing the differences in model implied covariance matrices is non-significant $\chi^{2}(74, \mathrm{~N}=2)=-10.89, p=1.0$, suggesting the models performed equally.

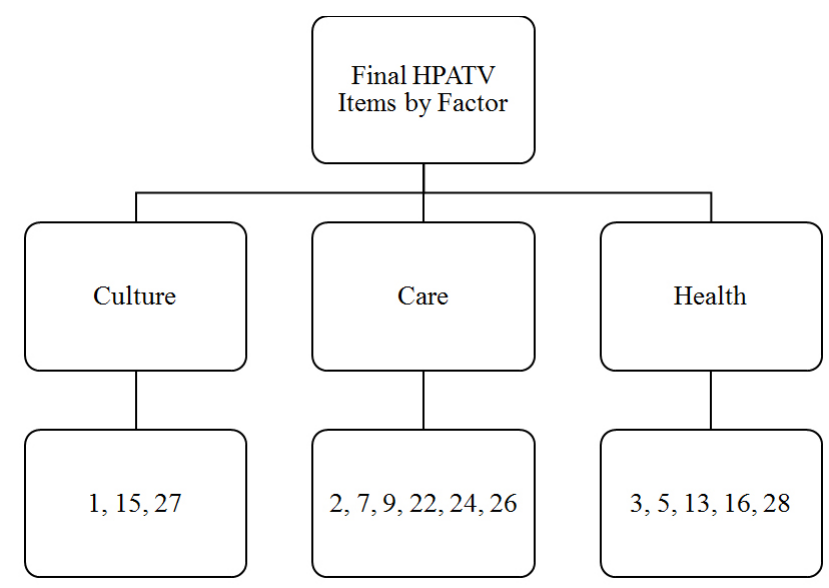

Figure 1. Final HPATV items by factor

\section{Discussion}

Attitudes-including beliefs, feelings, and behavioral intentions - are an important attribute of the healthcare equation that influence the quality of patient-provider interactions. ${ }^{[44]}$ The complexity of interactions with veteran patients can be compounded by possible intercultural differences between civilian and military cultures. ${ }^{[45]}$ Quality care for veterans is facilitated by health professionals' knowledge and confidence about delivering evidence-based services related to veteran health issues and culture. ${ }^{[15]}$ Adequate preparation, which may improve provider attitudes, is needed to recognize and respond to intercultural differences.

In order to provide patient-centered care to the veterans who receive services outside the VA system, community-based health professionals have a responsibility to screen regularly for military history, pursue training on the healthcare needs of veterans, and become familiar with veteran-specific resources for referrals. ${ }^{[1]}$ Within patient-provider interactions, certain communication techniques can be employed to help sensitively address the socio-cultural aspects of reverse culture shock that veterans may experience when readjusting to 
civilian life. ${ }^{[45]}$ Examples include eliciting challenges and yet explored how nurses and other health professionals view fostering growth across intrapersonal (e.g., mental and phys- this patient population and the associated responsibilities ical health), professional/educational (e.g., job and academic of tailoring care to veterans' needs. Understanding health satisfaction), and interpersonal domains (e.g., social support). students' and professionals' attitudes about caring for vetPre-service education on these topics would lay the foun- erans is an important first step in developing a healthcare dation for better quality care, and prior research has begun workforce that is knowledgeable and willing to serve this to identify core competencies in veterans health for nursing complex and growing patient population.

and other professions. ${ }^{[1,46]}$ However, prior research has not

Table 2. Standardized solution for wave 1 and 2 model estimation

\begin{tabular}{|c|c|c|c|c|c|}
\hline Factor & Item & Estimate Wave 1 & $p$ & Estimate Wave 2 & $p$ \\
\hline \multicolumn{6}{|c|}{ Factor Loadings } \\
\hline Culture & HPATV1 & 0.729 & .000 & 0.464 & .001 \\
\hline Culture & HPATV15r & 0.732 & .000 & 0.352 & .018 \\
\hline Culture & HPATV27r & 0.766 & .000 & 0.894 & .000 \\
\hline Care & HPATV2 & 0.651 & .000 & 0.842 & .000 \\
\hline Care & HPATV7 & 0.508 & .000 & 0.684 & .000 \\
\hline Care & HPATV9 & 0.610 & .000 & 0.745 & .000 \\
\hline Care & HPATV22 & 0.446 & .000 & 0.749 & .000 \\
\hline Care & HPATV24r & 0.726 & .000 & 0.319 & .024 \\
\hline Care & HPATV26r & 0.645 & .000 & 0.305 & .032 \\
\hline Health & HPATV3r & 0.420 & .001 & 0.189 & .228 \\
\hline Health & HPATV5 & 0.504 & .000 & 0.734 & .000 \\
\hline Health & HPATV13 & 0.930 & .000 & 0.813 & .000 \\
\hline Health & HPATV16 & 0.420 & .001 & 0.511 & .000 \\
\hline Health & HPATV28 & 0.581 & .000 & 0.143 & .369 \\
\hline \multicolumn{6}{|c|}{ Variances } \\
\hline- & HPATV1 & 0.468 & .000 & 0.785 & .000 \\
\hline- & HPATV15r & 0.464 & .000 & 0.876 & .000 \\
\hline- & HPATV27r & 0.413 & .000 & 0.201 & .481 \\
\hline- & HPATV2 & 0.576 & .000 & 0.290 & .004 \\
\hline- & HPATV7 & 0.742 & .000 & 0.532 & .000 \\
\hline- & HPATV9 & 0.627 & .000 & 0.444 & .000 \\
\hline- & HPATV22 & 0.801 & .000 & 0.438 & .000 \\
\hline- & HPATV24r & 0.473 & .000 & 0.898 & .000 \\
\hline- & HPATV26r & 0.583 & .000 & 0.907 & .000 \\
\hline- & HPATVЗr & 0.824 & .000 & 0.964 & .000 \\
\hline- & HPATV5 & 0.746 & .000 & 0.461 & .001 \\
\hline- & HPATV13 & 0.135 & .302 & 0.339 & .020 \\
\hline- & HPATV16 & 0.824 & .000 & 0.739 & .000 \\
\hline- & HPATV28 & 0.663 & .000 & 0.979 & .000 \\
\hline \multicolumn{6}{|c|}{ Latent Correlations } \\
\hline Culture & Care & 0.943 & .000 & 0.589 & .000 \\
\hline Culture & Health & 0.449 & .002 & 0.262 & .140 \\
\hline Care & Health & 0.697 & .000 & 0.675 & .000 \\
\hline
\end{tabular}

The HPATV provides a mechanism for assessing these at- subscale relate to awareness of and comfort with military titudes. Factor analysis of the instrument revealed 3 latent culture, largely cognitive and affective attitude components. factors: culture, care, and health. The items in the culture Items in the care subscale correspond with how health profes- 
sionals provide care to veteran patients, such as knowledge of and linkage with community-based services, screening for a history of military service, asking permission to discuss military-related health issues, and staying current on literature regarding veteran health issues; items in this subscale are largely about behavioral intentions. Finally, the health subscale items relate to knowledge and feelings toward prevalent veterans' health issues such as PTSD, military sexual trauma, and substance use; these are cognitive and affective attitude components.

The data generated by the HPATV and its 3 subscales has a number of potential applications that contribute to quality improvement. The HPATV can be used as a needs assessment tool in health professions schools and healthcare settings to identify areas of strength and weakness. Based on HPATV results, training curriculum can be developed or modified to increase positive feelings and confidence (affective component) and knowledge (cognitive component). Results could be used to better address the needs of veterans by guiding modification or development of organizational procedures and policies (behavioral component) to meet the needs of veteran patients. The HPATV can also be used as a program evaluation tool to determine the efficacy of veteran-specific education initiatives.

There are many directions for future research using the HPATV given its broad applicability across professions and settings. Studies could explore how health professionals' demographic characteristics, prior experience working with veterans, own veteran status, having veteran family members, and working in a VA versus community-based setting affect

\section{REFERENCES}

[1] McMillan LR, Crumbley D, Freeman J, et al. Caring for the veteran, military and family member nursing competencies: Strategies for integrating content into nursing school curricula. J Prof Nurs. 2017; 33(5): 378-386. PMid:28931486 https ://doi.org/10.1016/j. profnurs.2017.06.002

[2] Lee J, Sanders KM, Cox M. Honoring those who have served: How can health professionals provide optimal care for members of the military, veterans, and their families? Acad Med. 2014; 89(9): 11981200. PMid:24979290 https://doi .org/10.1097/ACM. 000000 0000000367

[3] Agha Z, Lofgren RP, VanRuiswyk JV, et al. Are patients at Veterans Affairs Medical Centers sicker?: A comparative analysis of health status and medical resource use. Arch Intern Med. 2000; 160(21): 32523257. PMid:11088086 https://doi .org/10.1001/archinte. 1 60.21 .3252

[4] Kline A, Falca-Dodson M, Sussner B, et al. Effects of repeated deployment to Iraq and Afghanistan on the health of New Jer- the culture, care, and health domains of their attitudes toward veteran patients. Additionally, a comparison of attitudes among different health disciplines could provide further insight into what type of pre-service training is of value for different professions.

\section{Conclusion}

As military conflicts continue worldwide and the veteran population expands and increasingly seeks care in communitybased settings, cultural competence of healthcare providers is paramount. Preparation of health professionals in the care of veterans should begin with an assessment of their attitudes toward this stigmatized and complex population with the goal of improving the quality of care provided. The HPATV was designed to capture nuances regarding health professionals' attitudes about providing care to veterans and identified three domains: culture, care, and health. Further research examining and understanding these domains and what factors might influence them, and building quality improvement initiatives based on these domains, will provide for sensitive, high quality, patient-centered care for veterans.

\section{ACKnOWledgements}

The authors wish to acknowledge Terri LaCoursiere Zucchero, $\mathrm{PhD}, \mathrm{RN}$, who contributed to item generation and the Delphi survey process. We also wish to acknowledge the subject matter experts who participated in the Delphi survey and item refinement, as well as the health professions students who piloted the scale.

\section{CONFLicts OF InTEREST Disclosure}

The authors declare that there is no conflict of interest. sey Army National Guard troops: Implications for military readiness. Am J Public Health. 2010; 100(2): 276-283. PMid:20019304 https://doi.org/10.2105/AJPH. 2009.162925

[5] Kazis LE, Miller DR, Clark J, et al. Health-related quality of life in patients served by the Department of Veterans Affairs: Results from the veterans health study. Arch Intern Med. 1998; 158: 626-632. https://doi.org/10.1001/archinte.158.6.626

[6] Koblinsky SA, Leslie LA, Cook ET. Treating behavioral health conditions of OEF/OIF Veterans and their families: A state needs assessment of civilian providers. Mil Behav Health. 2014; 2(2): 162172. PMid:24999446 https://doi.org/10.1080/21635781.2 014.890884

[7] Luby CD. Promoting military cultural awareness in an off-post community of behavioral health and social support service providers. Adv Soc Work. 2012; 13(1): 67-82.

[8] Tanielian T, Jaycox LH, Schell T, et al. Invisible wounds of war: Summary and recommendations for addressing psychological and cognitive injuries. Santa Monica, CA: The RAND Corporation; 2008. 
[9] Veterans Health Administration, Office of Rural Health. Strategic plan refresh: Fiscal years 2012-2014. Washington, DC: U.S. Department of Veterans Affairs; 2012.

[10] U.S. Department of Veterans Affairs. Veterans Access, Choice and Accountability Act of 2014 (“Choice Act”). Washington, DC: U.S. Department of Veterans Affairs; nd.

[11] Davis LL, Mahaney-Price AF, Tabb KD, et al. Alabama veterans rural health initiative: A preliminary evaluation of unmet health care needs. J Rural Soc Sci. 2011; 26(3): 14-31.

[12] Greedlinger R, Clervil R. Engaging veterans and families to enhance service delivery. Waltham, MA: National Center on Family Homelessness; 2011.

[13] Schell TL, Tanielian T, Farmer CM, et al. A needs assessment of New York State veterans. RAND Health Quarterly. 2011; 1(1): 14.

[14] Chretien JP, Chretien KC. Coming home from war. J Gen Intern Med. 2013; 28(7): 953-956. PMid:23435767 https://doi.org/10.1 007/s11606-013-2359-7

[15] Kilpatrick DG, Best CL, Smith DW, et al. Serving those who have served: Educational needs of health care providers working with military members, veterans, and their families. Charleston, SC: Medical University of South Carolina Department of Psychiatry, National Crime Victims Research and Treatment Center; 2011.

[16] Tanielian T, Jaycox LH (Eds.). Invisible wounds of war: Summary and recommendations for addressing psychological and cognitive injuries. Santa Monica, CA: The RAND Corporation; 2008.

[17] Association of American Medical Colleges. Serving those who serve America: Joining forces: Results of an AAMC survey. Washington, DC: Association of American Medical Colleges; 2012.

[18] Brener L, Von Hippel W, Kippax S, et al. The role of physician and nurse attitudes in the health care of injecting drug users. Subst Use Misuse. 2010; 45(7-8): 1007-1018. PMid:20441447 https: //doi.org/10.3109/10826081003659543

[19] Institute of Medicine. Crossing the quality chasm: A new health system for the 21st century. Washington, DC: The National Academies Press; 2001.

[20] Jeffreys MD, Leibowitz RQ, Finley E, et al. Trauma disclosure to health care professionals by veterans: Clinical implications. Mil Med. 2010; 175(10): 719-724. https://doi.org/10.7205/MILMED-D $-10-00054$

[21] Buck DS, Marconi Monteiro F, Kneuper S, et al. Design and validation of the health professionals' attitudes toward the homeless inventory. BMC Med Educ. 2005; 5(2): 1-8.

[22] Wang D. Social workers' attitudes toward older adults: A review of the literature. J Soc Work Educ. 2013; 49(1): 150-172.

[23] Wilson CK, West L, Stepleman L, et al. Attitudes toward LGBT patients among students in the health professions: Influence of demographics and discipline. LGBT Health. 2014; 1(3): 204-211. PMid:26789713 https://doi.org/10.1089/lgbt.2013.0016

[24] Center for Deployment Psychology. Self awareness exercise. Bethesda, MD: Uniformed Services University; nd.

[25] Eagly AH, Chaiken S. The psychology of attitudes. Fort Worth, TX: Harcourt Brace Jovanovich College Publishers; 1993.

[26] Maio G, Haddock G. The psychology of attitudes and attitude change. Los Angeles, CA: Sage; 2014.

[27] Zanna MP, Rempel JK. Attitudes: A new look at an old concept. In: Bar-Tal D, Kruglanski AW, eds. The social psychology of knowledge. Cambridge, England: Cambridge University Press; 1998: 315-334.
[28] Pratkanis AR, Breckler SJ, Greenwald AG., eds. Attitude structure and function. Hillsdale, NJ: Lawrence Erlbaum; 1989.

[29] Ajzen I, Fishbein M. Understanding attitudes and predicting social behavior. Englewood Cliffs, NJ: Prentice-Hall; 1980.

[30] Edwards D, Potter J. Discursive psychology, volume 8. Los Angeles, CA: Sage; 1992.

[31] Rinaca CA. The use of the tripartite model of attitudes to explain EMS providers' attitudes about the EMS agenda for the future [dissertation]. Norfolk, VA: Old Dominion University; 2005.

[32] University of Wisconsin Program Development \& Cooperative Extension. Collecting group data: Delphi technique. Madison, WI: University of Wisconsin; 2002.

[33] Klemenc-Ketis Z, Vrecko H. Development and validation of a professionalism assessment scale for medical students. Int J Med Educ. 2014; 5: 205-211. PMid:25382090 https://doi.org/10.5116/ ijme.544b.7972

[34] R Core Team. R: A language and environment for statistical computing. Vienna, Austria: R Foundation for Statistical Computing; 2017.

[35] Yves R. Lavaan: An R package for structural equation modeling. J Stat Softw. 2012; 48(2): 1-36.

[36] Epskamp S, Stuber S. semPlot: Path diagrams and visual analysis of various SEM packages' output. R package version 1.1; 2017.

[37] R Core Team. Foreign: Read data stored by Minitab, S, SAS, SPSS, Stata, Systat, Weka, dBase R package version 0.8-67; 2016.

[38] Revelle W. Psych: Procedures for Personality and Psychological Research. Evanston, IL: Northwestern University; 2017.

[39] Daróczi G, Tsegelskyi R. Pander: An R Pandoc writer. R package version 0.6.0.

[40] Guttman L. Some necessary and sufficient conditions for common factor analysis. Psychometrika. 1954; 19: 149-161. https: //doi.org/10.1007/BF02289162

[41] Kaiser HF. The application of electronic computers to factor analysis. Educ Psychol Meas. 1960; 20: 141-151. https ://doi .org/10.1 177/001316446002000116

[42] MacCallum RC, Browne MW, Sugawara HM. Power analysis and determination of sample size for covariance structure modeling. Psychol Methods. 1996; 1: 130-149. https://doi .org/10.1037/10 82-989X.1.2.130

[43] Heywood HB. On finite sequences of real numbers. Proc. R. Soc. Lond. A. 1931; 134(824): 486-501. https://doi.org/10.1098/ rspa. 1931.0209

[44] Halligan A. The importance of values in healthcare. J Royal Soc Med. 2008; 101(10): 480-481. PMid:18840859 https ://doi .org/10.1 258/jrsm.08k019

[45] Koenig CJ, Maguen S, Monroy JD, et al. Facilitating culture-centered communication between health care providers and veterans transitioning from military deployment to civilian life. Patient Education and Counseling. 2014; 95: 414-420. PMid:24742536 https: //doi.org/10.1016/j.pec.2014.03.016

[46] Moss JA, Moore RL, Selleck CS. Veteran competencies for undergraduate nursing education. Advances in Nursing Science. 2015; 38(4): 306-316. PMid:26517342 https://doi .org/10.1097/AN S. 0000000000000092 